\title{
Maximum Loadability Enhancement with a Hybrid Optimization Method
}

\author{
E. E. Hassan ${ }^{1}$, T. K. A. Rahman ${ }^{2}$, Z. Zakaria ${ }^{3}$, N. Bahaman ${ }^{4}$, M. H. Jifri ${ }^{5}$ \\ ${ }^{1,5}$ Faculty of Electrical Engineering, Universiti Teknikal Malaysia Melaka (UTeM), Malaysia \\ ${ }^{2}$ School of Science and Technology, Asia University, Wisma Subang Jaya, Selangor, Malaysia \\ ${ }^{3}$ Faculty of Electrical Engineering, Universiti Teknologi Mara, Shah Alam, Selangor, Malaysia \\ ${ }^{4}$ Faculty of Information and Communication Technology, University Teknikal Malaysia Melaka (UTeM), Malaysia
}

\begin{tabular}{l}
\hline \hline Article Info \\
\hline Article history: \\
Received March 26, 2018 \\
Revised Jul 25, 2018 \\
Accepted Aug 08, 2018 \\
\hline
\end{tabular}

Keywords:

ATBFO

Meta-heuristic evolutionary programming

RPP

SCRPP

\begin{abstract}
Nowadays, a power system is operating in a stressed condition due to the increase in demand in addition to constraint in building new power plants. The economics and environmental constraints to build new power plants and transmission lines have led the system to operate very close to its stability limits. Hence, more researches are required to study the important requirements to maintain stable voltage condition and hence develop new techniques in order to address the voltage stability problem. As an action, most Reactive Power Planning (RPP) objective is to minimize the cost of new reactive resources while satisfying the voltage stability constraints and labeled as Secured Reactive Power Planning (SCRPP). The new alternative optimization technique called Adaptive Tumbling Bacterial Foraging (ATBFO) was introduced to solve the RPP problems in the IEEE 57 bus system. The comparison common optimization Meta-Heuristic Evolutionary Programming and original Bacterial Foraging techniques were chosen to verify the performance using the proposed ATBFO method. As a result, the ATBFO method is confirmed as the best suitable solution in solving the identified RPP objective functions.
\end{abstract}

Copyright $@ 2018$ Institute of Advanced Engineering and Science. All rights reserved.

\section{Corresponding Author:}

E. E. Hassan,

Faculty of Electrical Engineering,

Universiti Teknikal Malaysia Melaka (UTeM), Malaysia.

Email: erwani@utem.edu.my

\section{INTRODUCTION}

Many countries have reported that millions of dollars were lost due to voltage collapse incidents. Failure to progress above the specific voltage magnitude leads to voltage collapse [1]. In other words, voltage collapse is due to voltage instability that refers to the inability of a power system to keep the steady state voltages at all buses [2]. Besides that, failure in congestion management may results in blackout of the whole or parts of power system. This situation is verified by a report which stated that an outage of a $345 \mathrm{kV}$ transmission line has caused blackout in Canada and U.S in August 2003 because the system is unable to sustain the additional load [3]. Therefore, efficient RPP planning would be able to avoid the occurrence of voltage collapse. Several objective functions were implemented in SCRPP in order to improve the voltage stability condition of a power system such as minimizing voltage deviation from specified operating points and maximizing static stability margin (SM) [4].

In order to obtain optimal solution to SCRPP, the efficient and reliable optimization technique has become necessary. These advanced and efficient solutions are able to overcome the weakness of the existing classical methods which are not capable to solve non convex, non-continuous and highly nonlinear solution such as in SCRPP problems [5]. Thus, today meta-heuristic optimization approaches such as Particle Swarming Optimization (PSO), Evolutionary Programming (EP), Genetic Algorithm (GA) and Bacterial 
Foraging Algorithm (BFA) with advanced search techniques make the problems possible to be solved. These techniques offered global optimal solutions, however, at the expense of computational time [6]. Therefore, recent researches are inspired to merge conventional methods and advanced optimization techniques for better and faster optimization approaches.

This study intended to introduce a new Adaptive Tumbling Bacterial Foraging Optimization (ATBFO) algorithm which is an improvement to the basic Bacterial Foraging Optimization (BFO) algorithm. The proposed technique was implemented to solve the single objective SCRPP problems. Finally, the performances of the newly developed technique ATBFO were compared with that provided by the EP and the basic BFO. The best solutions were identified based on the smallest total system losses and maximum loading point that the system can withstand. In addition, the aggregate function method was applied to confirm the outperformed method among them. The lowest total aggregate value is declared as the excellent approach for the SCRRP problem.

\section{SECURED REACTIVE POWER PLANNING}

RPP is also known as VAR planning in which reactive power sources are managed and planned optimally [7]. Reactive power can either inductive or capacitive in nature [8]. RPP is normally solved by using optimization methods. Various factors and objectives are taken into account in solving RPP in order to ensure for optimal power flow solution. The main objective of RPP is normally minimization of cost functions such as variable VAR cost, fixed VAR cost, real power losses and also fuel cost [9]. The authors in this reference also have explained on the deviation of the operating voltage from a specified voltage schedule and hence utilized Voltage Stability Margin (VSM). In Secured Reactive Power Planning (SCRPP), voltage stability criteria are normally treated as the constraints. Therefore, the importance of Load Margin (LM) assessment is used as a tool to indicate the maximum loading point in order to provide secure operating margin in power system operation.

\subsection{Load Margin Assessment}

Load Margin (LM) is broadly accepted in analyzing the closeness of the operating condition of a power system to its voltage collapse. The LM is defined as the quantity of load increment allowable before a power system reaches the unsecure voltage condition. The load margin was determined by gradually increase the load until the load flow failed to give solution.

The relationship between reactive power reserve and Voltage Stability Margin (VSM) was investigated by researchers in reference [10]. The authors in [11] proposed for re-dispatch of reactive power in order to improve the voltage stability condition of the power system. However, the total active power losses were not measured because they believed that the solution is not the optimum one. For that reason, many researchers have given attention to enhance voltage stability condition by sustaining the reactive power in a power system [12].

The important steps for load margin estimation that involved the load margin analysis and enhancement were discussed. Thus, load margin assessment can be classified into two categories in which the first is to forecast the MLP while the second one is to enhance the voltage stability margin for better stability condition.

\subsection{Objective Functions for SCRPP}

The consideration to be an objective function based on Maximum Loadability Point (MLP) improvement for all load busses in solving SCRPP and also at the improvement of MLP at the critical bus [13].

\subsubsection{Maximizing MLP}

MLP for a power networks is the maximum amount of load that could be sustained before it reached the unstable operating point. As referred to references [14], the LM or also called as VSM could be defined as the distance from the base case, $\lambda_{0}$ load to the maximum loading limit, $\lambda_{\max }$ prior to its imbalance point as shown in Figure 1. During the assessment, the weakest bus among the network and maximum load that it can sustain can also be determined. The bus with the smallest margin is identified as the weak or critical bus. This figure also illustrates the comparison between the MLP before optimizing the reactive power sources through RPP i.e point A and the MLP after the reactive sources are optimized i.e point B. 


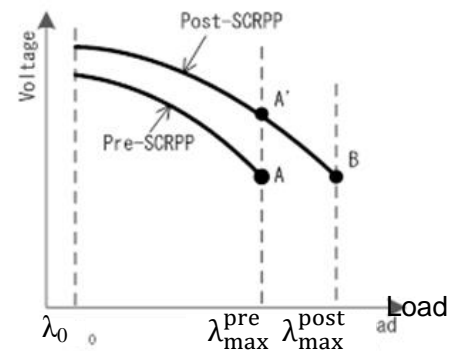

Figure 1. The comparison graph between pre and post SCRPP implementation

\subsubsection{Minimizing Total System Losses}

The objective function for total loss minimization is given by Equation 1 .

$$
\begin{aligned}
& \min f_{Q}=\sum_{k \in N_{G}} P_{k L o s s}(v, \theta)=\sum_{\substack{k \in N_{G} \\
k=(i, j)}} g_{k}\left(V_{i}^{2}+V_{j}^{2}-2 V_{i} V_{j} \cos \theta_{i j}\right) \mathrm{MW} \\
& V_{i_{\text {min }}} \leq V_{i} \leq V_{i_{\text {max }}} i \in N_{B} \\
& Q_{G i_{\text {min }}} \leq Q_{G i} \leq Q_{G i_{\text {Max }}} i \in\left\{N_{P V}, n_{s}\right\}
\end{aligned}
$$

where, $Q_{i}$ and $Q_{j}$ are reactive power at sending and receiving buses respectively, $Q_{G i}$ is generated reactive power of bus i, $V_{i}$ and $V_{j}$ are voltage magnitude at sending and receiving buses respectively. $P_{k L o s s,}$ is total active power loss over the network, $N_{B}$ is load bus, $N_{P V}$ is voltage controlled bus and $n_{s}$ is reference (slack) bus.

\subsubsection{The Important Control Variables}

The control variables considered are capacitor or reactor switching transformer tap changing [15] and active power of generator, to facilitate the requirement of SCRPP.

\section{METHODOLOGY}

\subsection{New Adaptive Bacterial Foraging Optimization (ATBFO) Algorithm}

This recent Bacterial Foraging Optimization (BFO) searching algorithm invented by K.M. Passino, is supported by the fact that natural selection tends to eliminate animals with poor foraging strategies against those with attractive foraging [16]. These poor hunters will be either eliminated or sometimes reshaped to good ones through a repeated generation process. Several processes of E. coli foraging that are present in our intestines are called chemotaxis, swarming, reproduction and elimination and dispersal [17]. Using the E.coli foraging strategy as in BFO, the global searching space is improved by modifying the tumbling approach by adapting the mutation technique applied in Meta-EP into tumbling expression implemented in basic BFO thus represented by new Equation 2 to 4 in ATBFO algorithm.

$$
\theta^{i}(j+1, k, l)=\theta^{i}(j, k, l)+C(i) \emptyset(i)
$$

Hence: $\emptyset(i)=\frac{\Delta(i)}{\sqrt{\Delta^{T}(i) \Delta(i)}}$, where $\Delta(i)=$ random vector for each bacterium, $\Delta^{T}(i)=$ transpose of random vector for each bacterium. Then, mutate the new position of Jlast by using given by Equation 2 .

$$
\begin{aligned}
& \emptyset^{\prime} i(j)=\emptyset(j) \exp \tau^{\prime} N(0,1)+\tau N i(0,1) \\
& P^{\prime} i(j)=P i(j)+\emptyset^{\prime} i(j) N j(0,1)
\end{aligned}
$$

where $\tau=\sqrt{\frac{1}{\sqrt{2 n}}}, \tau^{\prime}=\frac{1}{\sqrt{2 n}}, P^{\prime} i(j), P i(j), \emptyset^{\prime} i(j)$ and $\emptyset(j)$ is a $i^{\text {th }}$ component of respective vector, $N i(0,1)$ is normally distributed one dimensional random number with mean 0 and $1 . N j(0,1)$ indicates the random number will be new for each value of $j$. 


\subsection{A New ATBFO Algorithm for Single Objective Function SCRPP}

An intelligence heuristic technique named as ATBFO algorithm was implemented as an optimization mechanism for solving SCRPP problems with single objective solution. This single objective is either to maximize the Maximum Loadability Point (MLP) or minimize system losses while satisfying the operational constraints. The corresponding objective function is calculated while the value of the other is observed. The simulations were tested under tested on the IEEE 57 bus system for unstressed and stressed conditions as illustrated in Figure 2. The task also covered all possibilities of load increments as following:

a. Reactive load increment or Q increment

b. Real load increment or $\mathrm{P}$ increment and

c. Reactive and Real load increment or Q and P load increased simultaneously.

In addition, the ATBFO method was also executed on identified critical load bus growth called as Case 1. While, in Case 2 was when the load at all busses were increased simultaneously. During the implementation, different sizes of control variables were determined, such as Reactive Power Dispatch (RPD) $\mathrm{Q}_{\mathrm{gs}}$, Capacitor Placement (CP), $\mathrm{Q}_{\text {inj }}$ and Transformer Tap Change Setting (TTCS), $\mathrm{X}_{\mathrm{mer}}$. The solution in searching for optimal sizes of control variables were also categories into different group of RPP techniques such as $X_{\text {mer }}, Q_{i n j}, Q_{g s} \& Q_{i n j}, Q_{g s} \& X_{m e r}, Q_{i n j} \& X_{m e r}$ or $Q_{i n j}, Q_{g s} \& X_{m e r}$ as RPP technique respectively as referred in $[32,33]$. The overall implementations of the structure covered throughout the contribution were explained in depth by the subsequent Figure 2.

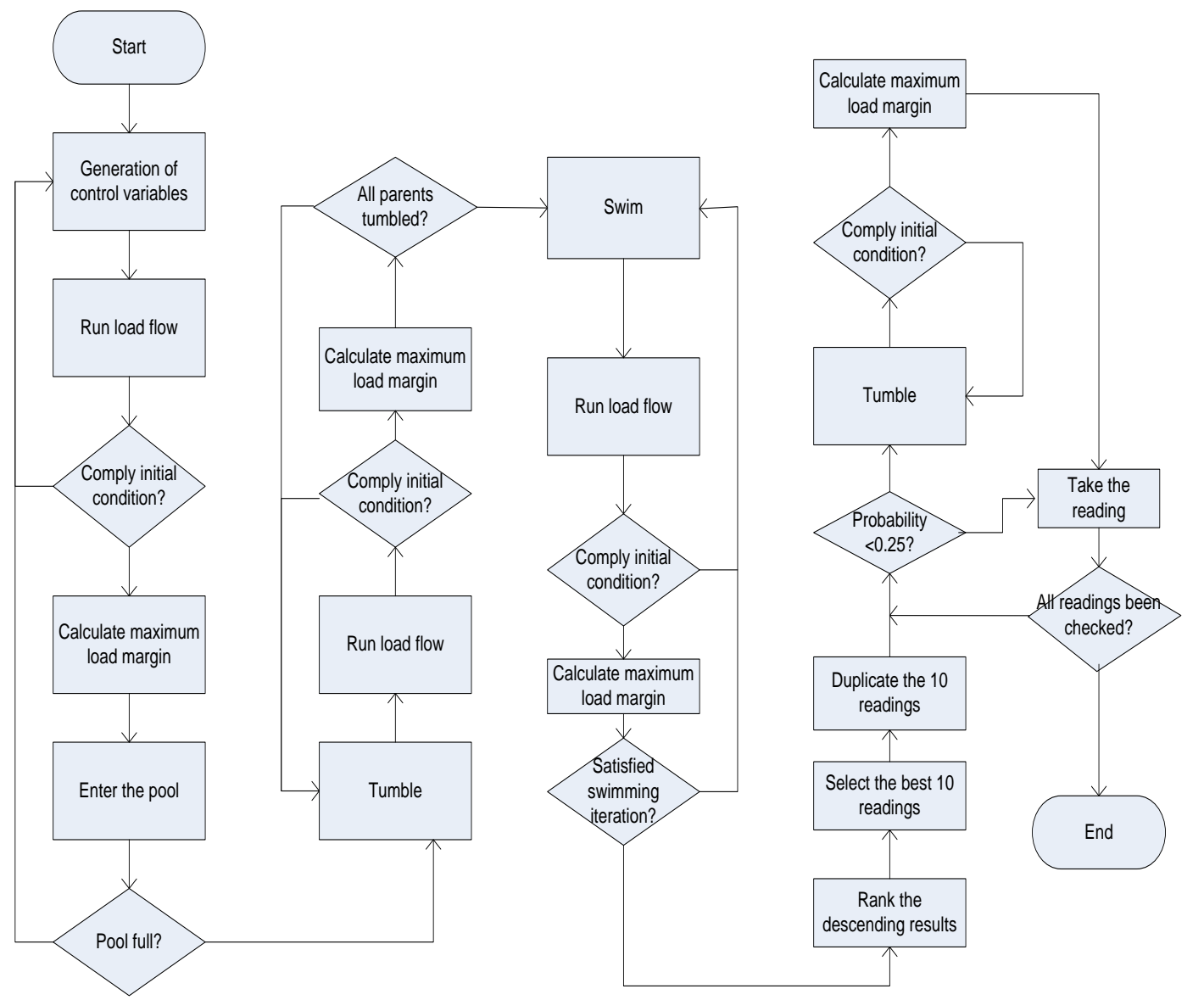

Figure 2. Flowchart of ATBFO process for SCRPP for stressed and unstressed condition

The proposed ATBFO was tested on the IEEE 57 bus system for each Single Objective SCRPP functions as the following:

a. SOSCRPP1=maximum MLP

b. SOSCRPP2=minimum total losses 
The similar optimization process using this ATBFO method which to minimize the total system losses SOSCRPP2 solutions were also obtained from Case 1 and Case 2 i.e during unstressed and stressed situations.

\subsection{Aggregate Function Method}

The aggregate function is introduced in this study as an alternative to describe the results obtained from optimization methods to meaningful evaluation and conclusion. From the results obtained, the least answers bring the smallest aggregate value among others objective functions and vice versa. At the end, the total aggregates are calculated and the smallest sum value as the finest solution.

\section{RESULTS AND DISCUSSION}

This section discusses the comparison between two individual objective functions namely SOSCRPP1 and SOSCRPP2 which are to maximize the MLP and to minimize the total losses. Table 1 shows the improved voltages and their corresponding losses after the implementation SCRPP by optimizing RPD+TTCS+CP using ATBFO (Point A'). Similarly, the less total loss was determined from SOSCRPP1 as compared to SOSCRPP2 at the same Point A'. Initially, the Pre-SCRPP (Point A) has 0.849V (Vmin), 30.4575MW (Losses) and 195\% (MLP).

Table 1. Comparison between SOSCRPP1 and SOSCRPP2 at Point A' (After the Implementation of SCRPP) for Case 1

\begin{tabular}{|c|c|c|c|c|c|}
\hline \multicolumn{6}{|c|}{ Single objective of SCRPP for Case 1 using (RPD+TTCS+CP) technique at Point A' } \\
\hline \multirow{9}{*}{ 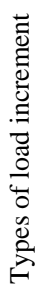 } & Objective function & SOSCRPP 1 & SOSCRPP 2 & SOSCRPP 1 & SOSCRPP 2 \\
\hline & & Minimum & Minimum & Losses & Losses \\
\hline & & Voltage, (p.u) & Voltage, (p.u) & $(\mathrm{MW})$ & $(\mathrm{MW})$ \\
\hline & P load-unstressed condition & 0.957 & 0.877 & 31.2383 & 31.9231 \\
\hline & P load-stressed condition & 0.940 & 0.912 & 30.9819 & 31.8038 \\
\hline & Q load-unstressed condition & 0.971 & 0.866 & 28.2897 & 28.6808 \\
\hline & Q load-stressed condition & 0.973 & 0.942 & 27.9983 & 27.9994 \\
\hline & Q \& P load-unstressed condition & 0.948 & 0.885 & 29.5578 & 30.1719 \\
\hline & Q \& P load-stressed condition & 0.951 & 0.885 & 29.2530 & 30.1169 \\
\hline
\end{tabular}

Table 1 highlights that SOSCRPP 1 resulted in the highest minimum voltage improvement for all types of load increments at the critical load bus 31. The SOSCRPP1 is solved through the improved ATBFO which optimized the RPD+RPP+CP with minimizing total losses and maximizing MLP as objective functions.

While in case 2, the results obtained from SOSCRPP1 (objective function: maximizing MLP) and SOSCRPP2 (objective function: minimizing total losses) for P load, Q load and Q with P load increments during the unstressed and stressed situations are compared as shown in Table 2. The table also tabulates the minimum voltages after of the implementation of SCRPP.

Table 2. Comparison between SOSCRPP1 and SOSCRPP2 at Point A' (post optimization)for Case 2

\begin{tabular}{|c|c|c|c|c|c|}
\hline \multirow{10}{*}{ 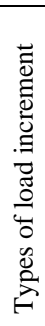 } & \multicolumn{4}{|c|}{ Single objective of SCRPP for Case 2 using (RPD+TTCS+CP) technique } & \multirow{4}{*}{$\begin{array}{c}\text { SOSCRPP2 } \\
\text { Losses } \\
\text { (MW) }\end{array}$} \\
\hline & Objective function & SOSCRPP1 & SOSCRPP2 & SOSCRPP1 & \\
\hline & & Minimum & Minimum & Losses & \\
\hline & & Voltage, (p.u & Voltage, (p.u) & (MW) & \\
\hline & P load-unstressed condition & 0.931 & 0.906 & 70.6513 & 71.6664 \\
\hline & P load-stressed condition & 0.935 & 0.898 & 66.4320 & 67.7000 \\
\hline & Q load-unstressed condition & 0.932 & 0.919 & 29.3769 & 29.7674 \\
\hline & Q load-stressed condition & 0.924 & 0.913 & 29.9849 & 29.7363 \\
\hline & Q \& P load-unstressed condition & 0.925 & 0.899 & 48.2148 & 48.5307 \\
\hline & Q \& P load-stressed condition & 0.939 & 0.887 & 46.4769 & 46.6924 \\
\hline
\end{tabular}

The results gained from SOSCRPP1 show higher minimum voltage as compared to that obtained by SOSCRPP2. In addition, SOSCRPP 1 also leads to lower total losses. Hence, SOSCRPP1 is better in performance as compared to SOSCRPP2 for Case 1 and Case 2. 


\subsection{Comparison of Single Objective Function in SCRPP among Optimization Techniques}

The single objective results for maximizing MLP obtained by ATBFO were compared with those obtained from the original BFO and Meta-EP approaches. Thus, Table 3 highlights the comparison of the results obtained after solving SCRPP using the above approaches i.e at Point A' and Point B.

Aggregate function was introduced in the comparative study in order to identify the technique which gives the best optimization performance as in Table 4. At Point A', the observed performances are the minimum voltage improvement and total losses minimization. While at point B, MLP enhancement is observed.

In Table 4, the performance of each optimization technique is ranked and value 1 is given to the best result, while value 3 is given to the worst. The least total aggregate indicates the best performance overall. From this table, it shows that ATBFO always resulted in the best overall performance. Hence, it can be concluded that ATBFO outperformed the other two optimization technique. This conclusion is summarized in Table 5.

Therefore, the outstanding optimization computational tool is recorded by the new ATBFO, followed by Meta-EP and finally the original BFO algorithm.

Table 3. Comparison between ATBFO and Others Optimization Techniques for SOSCRPP1

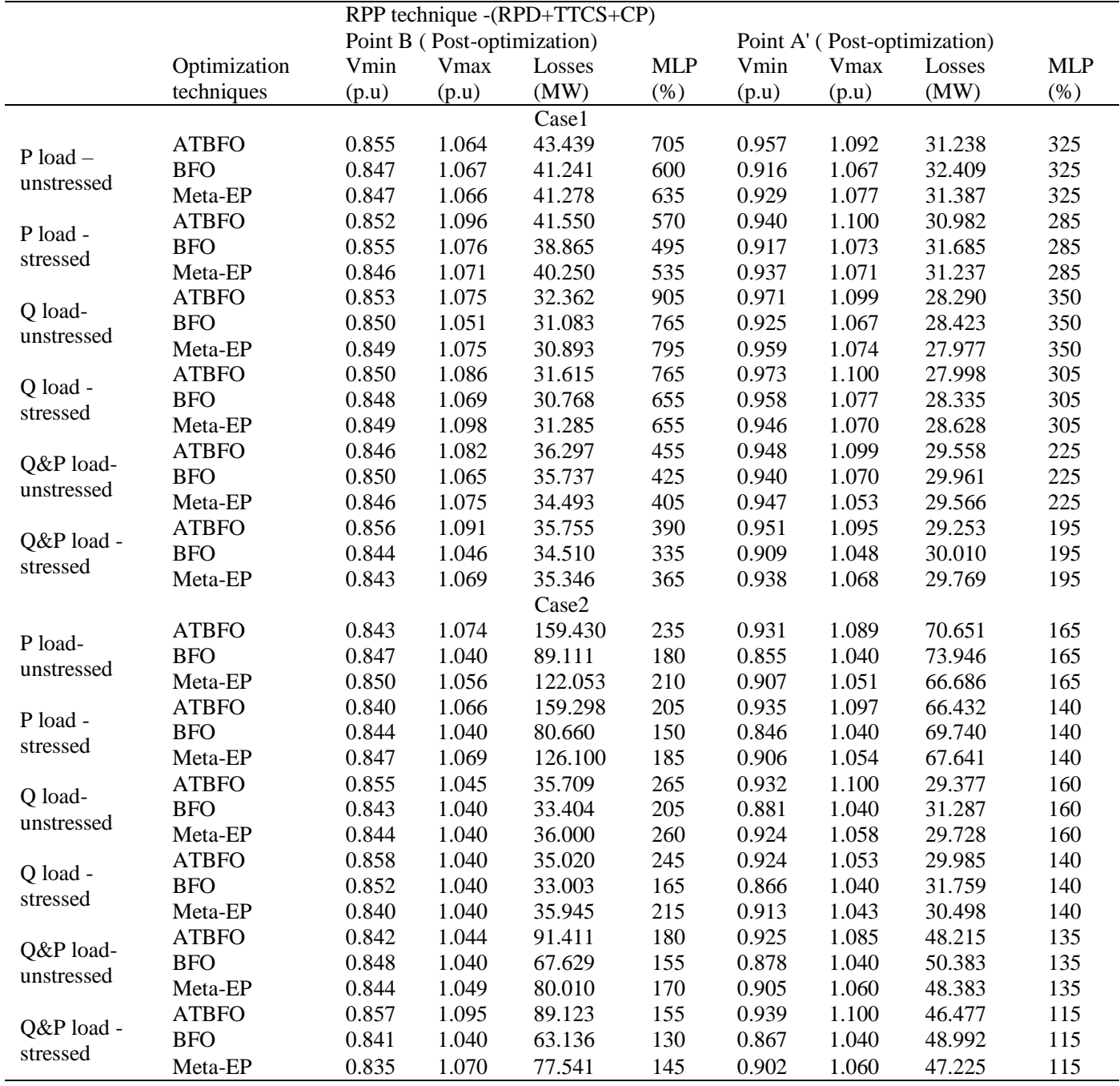


Table 4. Comparison between ATBFO and Others Optimization Techniques for SOSCRPP1 Using Aggregate Performance

\begin{tabular}{|c|c|c|c|c|c|}
\hline \multicolumn{6}{|c|}{ Aggregate Function } \\
\hline & \multirow[b]{2}{*}{ Optimization techniques } & \multicolumn{2}{|c|}{ Point A } & \multirow{2}{*}{$\begin{array}{c}\text { Point B } \\
\text { MLP }\end{array}$} & \multirow[b]{2}{*}{ Total Aggregates } \\
\hline & & Vmin & Losses & & \\
\hline \multicolumn{6}{|c|}{ Case1 } \\
\hline \multirow{3}{*}{ P load-unstressed } & ATBFO & 1.0 & 1.0 & 1.0 & 3.0 \\
\hline & BFO & 3.0 & 3.0 & 3.0 & 9.0 \\
\hline & Meta-EP & 2.0 & 2.0 & 2.0 & 6.0 \\
\hline \multirow{3}{*}{ P load -stressed } & ATBFO & 1.0 & 1.0 & 1.0 & 3.0 \\
\hline & BFO & 3.0 & 3.0 & 3.0 & 9.0 \\
\hline & Meta-EP & 2.0 & 2.0 & 2.0 & 6.0 \\
\hline \multirow{3}{*}{ Q load- unstressed } & ATBFO & 1.0 & 2.0 & 1.0 & 4.0 \\
\hline & BFO & 3.0 & 3.0 & 3.0 & 9.0 \\
\hline & Meta-EP & 2.0 & 1.0 & 2.0 & 5.0 \\
\hline \multirow{3}{*}{ Q load -stressed } & ATBFO & 1.0 & 1.0 & 1.0 & 3.0 \\
\hline & BFO & 2.0 & 2.0 & 2.0 & 6.0 \\
\hline & Meta-EP & 3.0 & 3.0 & 3.0 & 9.0 \\
\hline \multirow{3}{*}{ Q\&P load-unstressed } & ATBFO & 1.0 & 2.0 & 1.0 & 4.0 \\
\hline & BFO & 3.0 & 3.0 & 2.0 & 8.0 \\
\hline & Meta-EP & 2.0 & 1.0 & 3.0 & 6.0 \\
\hline \multirow{3}{*}{ Q\&P load -stressed } & ATBFO & 1.0 & 1.0 & 1.0 & 3.0 \\
\hline & BFO & 3.0 & 3.0 & 3.0 & 9.0 \\
\hline & Meta-EP & 2.0 & 2.0 & 2.0 & 6.0 \\
\hline \multicolumn{6}{|c|}{ Case2 } \\
\hline \multirow{3}{*}{ P load-unstressed } & ATBFO & 1.0 & 2.0 & 1.0 & 4.0 \\
\hline & BFO & 3.0 & 3.0 & 3.0 & 9.0 \\
\hline & Meta-EP & 2.0 & 1.0 & 2.0 & 5.0 \\
\hline \multirow{3}{*}{ P load -stressed } & ATBFO & 1.0 & 1.0 & 1.0 & 3.0 \\
\hline & BFO & 3.0 & 3.0 & 3.0 & 9.0 \\
\hline & Meta-EP & 2.0 & 2.0 & 2.0 & 6.0 \\
\hline \multirow{3}{*}{ Q load-unstressed } & ATBFO & 1.0 & 1.0 & 1.0 & 3.0 \\
\hline & BFO & 3.0 & 3.0 & 3.0 & 9.0 \\
\hline & Meta-EP & 2.0 & 2.0 & 2.0 & 6.0 \\
\hline \multirow{3}{*}{ Q load -stressed } & ATBFO & 1.0 & 1.0 & 1.0 & 3.0 \\
\hline & BFO & 3.0 & 3.0 & 3.0 & 9.0 \\
\hline & Meta-EP & 2.0 & 2.0 & 2.0 & 6.0 \\
\hline \multirow{3}{*}{ Q\&P load-unstressed } & ATBFO & 1.0 & 1.0 & 1.0 & 3.0 \\
\hline & BFO & 3.0 & 3.0 & 3.0 & 9.0 \\
\hline & Meta-EP & 2.0 & 2.0 & 2.0 & 6.0 \\
\hline \multirow{3}{*}{ Q\&P load -stressed } & ATBFO & 1.0 & 1.0 & 1.0 & 3.0 \\
\hline & BFO & 3.0 & 3.0 & 3.0 & 9.0 \\
\hline & Meta-EP & 2.0 & 2.0 & 2.0 & 6.0 \\
\hline
\end{tabular}

Table 5. Comparison between ATBFO and Others Optimization Techniques for SOSCRPP1 for Overall Performance

\begin{tabular}{cccc}
\hline Optimization Techniques & ATBFO & BFO & MetaEP \\
\hline & Case1 & & \\
P load-unstressed & 3.0 & 9.0 & 6.0 \\
P load -stressed & 3.0 & 9.0 & 6.0 \\
Q load- unstressed & 4.0 & 9.0 & 5.0 \\
Q load -stressed & 3.0 & 6.0 & 9.0 \\
Q\&P load-unstressed & 4.0 & 8.0 & 6.0 \\
Q\&P load -stressed & 3.0 & 9.0 & 6.0 \\
& Case2 & & \\
P load-unstressed & 4.0 & 9.0 & 5.0 \\
P load -stressed & 3.0 & 9.0 & 6.0 \\
Q load- unstressed & 3.0 & 9.0 & 6.0 \\
Q load -stressed & 3.0 & 9.0 & 6.0 \\
Q\&P load-unstressed & 3.0 & 9.0 & 6.0 \\
Q\&P load -stressed & 3.0 & 9.0 & 6.0 \\
Overall Aggregates & 39.0 & 104.0 & 73.0 \\
\hline
\end{tabular}

\section{CONCLUSION}

The objective of SCRPP was to maximize the MLP. In other words, the system has the capability to support extra loads before going into the voltage instability point. Hence, the number of voltage collapse events could be reduced. The MLP considered in the study were P, Q and P \& Q load increases, while two cases were analyzed, which were MLP at the critical bus (case 1) and MLP for all load buses simultaneously (case 2). Single objective functions namely, total losses minimization and MLP improvement were 
implemented and analyzed in solving the SCRPP problems. Several RPP approaches were studied and it was found that optimizing RPD, CP and TTCS simultaneously gave the best results. Hence, ATBFO was utilized in SCRPP in order to optimize the RPD, CP and TTCS simultaneously so that the required optimal results would be obtained. The performance ATBFO was compared with that obtained by BFO and Meta-EP. Based on the analysis, it was found that ATBFO performed better in terms of MLP improvement, minimum voltage improvement and total losses minimization.

\section{ACKNOWLEDGEMENT}

We thank you to Universiti Teknikal Malaysia Melaka (UTeM) and Kementerian Pengajian Tinggi (KPT) by funding this research paper successful through the grant of RAGS/1/2015/TK0/ FKE/03/B00094.

\section{REFERENCES}

[1] C.M. Affonso, L.C.P. da Silva, F.G.M. Lima, S. Soares. Optimal MW/MVAR dispatch and minimal load shedding strategy for improving voltage stability margin. Power Engineering Society General Meeting, 2003, IEEE, 2003. 2: 895.

[2] A. Laifa, M. Boudour. Facts allocation for power systems voltage stability enhancement using MOPSO. Systems, Signals and Devices, 2008. IEEE SSD 2008. 5th International Multi-Conference on, 2008. 1-6.

[3] E.E Hassan, Z. Zakaria, T.K.A. Rahman. Improved Adaptive Tumbling Bacterial Foraging Optimization (ATBFO) for emission constrained economic dispatch problem. Proceedings of the World Congress on Engineering. 2012; 2 : $1-4$.

[4] F.I.H. Hassim, I. Musirin, T.K.A. Rahman. Voltage Stability Margin Enhancement Using Evolutionary Programming (EP). Research and Development, 2006. SCOReD 2006. 4th Student Conference on, 2006. 2006; 235-240.

[5] W. Yurong, L. Fangxing, W. Qiulan, C. Hao. Reactive Power Planning Based on Fuzzy Clustering, Gray Code, and Simulated Annealing. Power Systems, IEEE Transactions. 2011; 26: 2246-2255.

[6] S. Mishra. Hybrid least-square adaptive bacterial foraging strategy for harmonic estimation. Generation, Transmission and Distribution, IEE Proceedings-. 2005; 152: 379-389.

[7] Z. Wenjuan, L. Fangxing, L.M. Tolbert. Voltage stability constrained optimal power flow (VSCOPF) with two sets of variables (TSV) for reactive power planning. Transmission and Distribution Conference and Exposition, 2008. 2008; $1-6$.

[8] H. Barot, K. Bhattacharya. Optimal Reactive Power Planning and Compensation Effects on Transmission Loss Components.Power Engineering Society General Meeting, IEEE. 2007; pp. 1-7.

[9] Z. Wenjuan, L. Fangxing, L.M. Tolbert. Review of Reactive Power Planning: Objectives, Constraints, and Algorithms. Power Systems, IEEE Transactions. 2007; 22: 2177-2186.

[10] B. Lixin, H. Zhenyu, X. Wilsun. Online voltage stability monitoring using VAR reserves. Power Systems, IEEE Transactions. 2003; 18: 1461-1469.

[11] M. Yao, J.L. Mathieu, D. K. Molzahn. Using demand response to improve power system voltage stability margins. 2017 IEEE Manchester Power Tech. 2017; 1-6.

[12] J. T. Ma, L.L. Lai. Evolutionary programming approach to reactive power planning. Generation, Transmission and Distribution, IEE Proceedings-.1996; 143: 365-370.

[13] N. Aminudin, T.K.A. Rahman, I. Musirin. Optimal Power Flow for Load Margin Improvement using Evolutionary Programming.Research and Development, 2007. SCOReD 2007. 5th Student Conference on, 2007; 1-6.

[14] N. Aminudin, T.K.A. Rahman, I. Musirin. Optimal reactive Power Planning for Load margin Improvement using Multi Agent Immune EP. 2010 Ieee World Congress On Computational Intelligence, 2010; 2010.

[15] L.L. Garver, "Transmission Network Estimation Using Linear Programming," Power Apparatus and Systems, IEEE Transactions on, vol. PAS-89, pp. 1688-1697, 1970.

[16] Z. Zakaria, T.K.A. Rahman, E.E. Hassan. Economic load dispatch via an improved Bacterial Foraging Optimization. Power Engineering and Optimization Conference (PEOCO), 2014 IEEE 8th International. 2014; 380385.

[17] W. Zhao, L. Wang. An effective bacterial foraging optimizer for global optimization. Information Sciences. 2016; 329: 719-735. 